\title{
ON THE POLYHEDRAL SCHOENFLIES THEOREM
}

\author{
M. L. CURTIS $^{1}$ AND E. C. ZEEMAN
}

In this note we observe a relationship between the polyhedral Schoenflies problem and the question of whether the double suspension $M^{5}$ of a Poincare manifold is the 5 -sphere. In particular we show that if $M^{5}=S^{5}$, then a polyhedral embedding of $S^{n-1}$ in $S^{n}$ must be very "nice" if the Schoenflies theorem is to hold.

Definition 1. Let $\Delta^{n}$ be the standard $n$-simplex and $\dot{\Delta}^{n}$ be its boundary. A finite simplicial complex $K$ is a combinatorial $n$-sphere if there exists a piecewise linear homeomorphism $h: K \rightarrow \dot{\Delta}^{n+1}$.

Definition 2. An embedding $S^{n-1} C S^{n}$ is nice if there is a simplicial decomposition of $S^{n}$ such that $S^{n-1}$ is a subcomplex and both $S^{n-1}$ and $S^{n}$ are combinatorial spheres.

We have the following theorem (see [5]):

Theorem 1. If the embedding $S^{n-1} \subset S^{n}$ is nice, then the Schoenflies theorem holds; i.e., $S^{n}-S^{n-1}$ consists of two disjoint $n$-cells.

Definition 3. An embedding $S^{n-1} \subset S^{n}$ is of type I if $S^{n}$ can be represented as a combinatorial $n$-sphere with $S^{n-1}$ a subcomplex. An embedding is of type II if there is a simplicial decomposition of $S^{n}$ such that $S^{n-1}$ is a subcomplex which is a combinatorial $(n-1)$ sphere.

We construct a definite Poincaré manifold $M^{3}$ in $S^{4}$. Let $P$ be the 2-polyhedron obtained by attaching the boundaries of two disks to two oriented curves $a$ and $b$ (with one common point) according to the formulae $a^{-2} b a b=1, b^{-5} a b a b=1$. Then $\pi_{1}(P)$ has the presentation $\left\{a, b \mid a^{-2} b a e=1, b^{-5} a b a b=1\right\}$, and Newman [4] has shown that $\pi_{1}(P) \neq 0$. Now $P$ can be embedded in $S^{4}$ as a subcomplex (see [2; $4]$ ) with $S^{4}$ decomposed as a combinatorial 4 -sphere. Then the boundary $M^{3}$ of a nice neighborhood of $P$ is a Poincaré manifold [2]. It follows that the double suspension $M^{5}$ of $M^{3}$ is a subcomplex of the combinatorial 6-sphere $S^{6}$. This is used in Theorem 3 .

We note that if $M^{5}$ is locally euclidean, then $M^{5}=S^{5}$. For Mazur [3] has proved that if $X$ is a finite polyhedron and the cone $C(X)$ is locally $k$-euclidean at the cone point, then $C(X)-X=E^{k}$. Now $M^{5}$ is the suspension of the single suspension $M^{4}$ of $M^{3}$ and the suspension with one suspension point removed is just $C\left(M^{4}\right)-M^{4}$. If this 1960.

Presented to the Society, September 2, 1960; received by the editors February 5,

${ }^{1}$ Senior NSF Fellow. 
is $E^{5}$, then the suspension is just the 1-point compactification of $E^{5}$, namely $S^{5}$.

ThEOREM 2. If $M^{5}=S^{5}$, then the Schoenflies theorem fails for embeddings of type II with $n=5$.

Proof. Let $\sigma$ be a 3 -simplex of $M^{3}$ with boundary $\beta$. Then the double suspension of $\beta$ is a combinatorial 4-sphere $S^{4}$ in $M^{5}=S^{5}$. But $\pi_{1}\left(M^{3}-\sigma\right)=\pi_{1}\left(M^{3}\right) \neq 0$ and one complementary domain of $S^{4}$ in $S^{5}$ is just $\left(M^{3}-\sigma\right) \times I$, which is not simply connected.

Theorem 3. If $M^{5}=S^{5}$, then the Schoenflies theorem fails for embeddings of type I with $n=6$.

Proof. By the construction of $M^{3} C S^{4}$ given above, we have that $M^{5}=S^{5} C S^{6}$ is an embedding of type I with $n=6$. Let $D^{3}$ be the complementary domain of $M^{3}$ in $S^{4}$ which contains $P$. By projecting from suspension points we can get deformation retractions of a complementary domain $D^{5}$ (of $S^{5} \subset S^{6}$ ) onto $D^{4}$ (of $M^{4} C S^{5}$ ) onto $D^{3}$. Hence $D^{5}$ is not simply connected and therefore is not a cell.

REMARK. Since it seems difficult to prove that $M^{5} \neq S^{5}$, it must be hard to show that embeddings of types I and II satisfy the simple condition $S^{n-1} \times I \subset S^{n}$ which Morton Brown [1] has shown is a necessary and sufficient hypothesis for the Schoenflies theorem.

\section{REFERENCES}

1. Morton Brown, Outline of a proof of the generalised Schoenfies theorem, Bull. Amer. Math. Soc. vol. 66 (1960) pp. 74-76.

2. M. L. Curtis and R. L. Wilder, The existence of certain types of manifolds, Trans. Amer. Math. Soc. vol. 91 (1959) pp. 152-160.

3. Barry Mazur, On embeddings of spheres, Bull. Amer. Math. Soc. vol. 65 (1959) pp. 59-65.

4. M. H. A. Newman, Boundaries of ULC sets in Euclidean space, Proc. Nat. Acad. Sci. U.S.A. vol. 34 (1948) pp. 193-196.

5. —_, The division of Euclidean space by spheres, Proc. Roy. Soc. London, to appear.

Florida State University AND

Gonville and Caius College, Cambridge, England 